\title{
Preliminary study of the use of E6/E7mRNA detection in screening and triage management of HR-HPV infection during pregnancy
}

\author{
Yong-Mei Yang ${ }^{1}$, Shi-Jun Wang ${ }^{1}$, Feng-Ying Wang ${ }^{1}$, Rui Chen ${ }^{2}$, Qun Xiao ${ }^{1}$, Ning Kang ${ }^{1}$, \\ Qing-Ping Liao ${ }^{1,2}$ \\ ${ }^{1}$ Department of Obstetrics and Gynecology, Xuanwu Hospital, Capital Medical University, Beijing, China; ${ }^{2}$ Department of Obstetrics and \\ Gynecology, Beijing Tsinghua Changgung Hospital, Beijing, China \\ Contributions: (I) Conception and design: YM Yang, QP Liao; (II) Administrative support: QP Liao; (III) Provision of study materials or patients: SJ \\ Wang, Q Xiao, N Kang; (IV) Collection and assembly of data: FY Wang, Q Xiao, N Kang; (V) Data analysis and interpretation: SJ Wang, R Chen; (VI) \\ Manuscript writing: All authors; (VII) Final approval of manuscript: All authors. \\ Correspondence to: Dr. Qing-Ping Liao. Department of Obstetrics and Gynecology, Xuanwu Hospital, Capital Medical University, Beijing, China. \\ Email: liao_qinping@126.com.
}

Background: Detection of E6 and E7 mRNA load of high-risk human papillomavirus (HR-HPV) infection during pregnancy was compared with conventional cytopathology and DNA detection by pathological examination as colposcopy to evaluate the application of E6 and E7 mRNA detection in the diagnosis and management in HR-HPV infection for high -grade cervical lesions during pregnancy.

Methods: From January 2014 to June 2019, 1,058 pregnant women of childbearing age who were filed for regular obstetrics in the Department of Obstetrics and Gynecology, Xuanwu Hospital of Capital Medical University, were separately assessed using cervical liquid-based cytology and HPV DNA detection. If the results were abnormal, colposcopy was performed as a follow-up. The presence of HR-HPV E6/E7 mRNA fragments was detected through the HR-HPV E6/E7 mRNA test, and monitored at the same time as colposcopy. The diagnostic efficacy of the HR-HPV DNA test versus the HR-HPV E6/E7 mRNA test for high-grade cervical lesions during pregnancy was compared.

Results: The positive rate of HR-HPV E6/E7 mRNA detection in the overall cervical intraepithelial neoplasia (CIN) and above during pregnancy was lower than that with HR-HPV DNA detection, and there was a significant statistical difference between the two methods. In CIN I and normal or inflammatory results, the positive rate of HR-HPV E6/E7 mRNA detection was lower than that of HR-HPV DNA detection, while in the results of CIN II and CIN III, the positive rate of the two was not significantly different. HR-HPV E6/E7 mRNA detection is the same as HR-HPV DNA detection, both of which increased with the severity of cervical lesions, and the positive rate increased. In cases of maintenance or progression of cervical lesions, the positive rate of HR-HPV E6/E7 mRNA detection during pregnancy can reach $81.8 \%$. High-grade cervical lesions during pregnancy had a higher rate of reversal to a lower level after delivery.

Conclusions: The results suggested that the use of HR-HPV E6/E7 mRNA detection in cases of positive HR-HPV DNA detection can significantly improve the diagnostic specificity of CIN II and above highgrade cervical lesions.

Keywords: E6 and E7 mRNA detection; human papillomavirus infection (HPV infection); cervical lesions; pregnancy

Submitted Jul 06, 2021. Accepted for publication Sep 03, 2021.

doi: 10.21037/atm-21-4026

View this article at: https://dx.doi.org/10.21037/atm-21-4026 


\section{Introduction}

The occurrence of cervical cancer and persistent infection of high-risk human papillomavirus (HR-HPV) are closely related (1). Almost all cervical cancers are induced by HRHPV infection, mainly HPV16 and HPV18 (2). In most cases of HPV infection, the immune system can clear the $\mathrm{HPV}$ virus. But, persistent infection of HPV occurs in $10-15 \%$ of cases, and about $1 \%$ of HR-HPV infections finally develops into cervical cancer (3). During cancer transformation involving the viral infection site and changes in cervical cell morphology, integration of viral and host DNA to achieve reverse transcription is considered an important sign of the malignant progression of cervical lesions $(4,5)$.

Detecting HPV DNA has high clinical sensitivity but low specificity. A positive test result cannot accurately determine whether there are high-grade cervical histological lesions, which can lead to overtreatment and wasting of medical resources (6). Diagnosis and treatment of cervical lesions during pregnancy needs to consider the effect on both the pregnant woman and the fetus, requiring extra precautions. HR-HPV infection, if it is unclear whether there are cervical lesions, can cause unnecessary mental stress on the pregnant woman or the diagnosis of cervical cancer may be overlooked. Persistent infection of HRHPV initiates the carcinogenic process by upregulation of HPV E6/E7 protein expression, and overexpression of HPV E6/E7 protein is closely associated with the risk of cervical lesions progression. In non-pregnant women, E6 and E7 mRNA testing has the same clinical sensitivity and negative predictive value as DNA testing, while significantly improving the specificity of the test and reducing the false positive rate (7-9). However, whether E6/E7mRNA detection can be used for the diagnosis and management of HPV infection during pregnancy is untested. In this study, we compared detection of the E6/E7 mRNA load of HRHPV infection during pregnancy with DNA detection. The pathological results of cervical biopsy were used as the standard to explore the application of E6/E7 mRNA detection in the diagnosis of HPV infection and cervical lesions during pregnancy. It was the first time for our work to find that in cases of maintenance or progression of cervical lesions, the positive rate of HR-HPV E6/E7 mRNA detection during pregnancy can reach $81.8 \%$. Additionally, the use of HR-HPV E6/E7 mRNA detection in cases of positive HR-HPV DNA detection can significantly improve the diagnostic specificity of CIN II and above high-grade cervical lesions. Improved triage improves diagnostic efficiency, thereby reducing unnecessary invasive examinations during pregnancy. We present the following article in accordance with the STARD reporting checklist (available at https://dx.doi.org/10.21037/atm-21-4026).

\section{Methods}

\section{Research subjects}

Pregnant women of childbearing age who were filed for regular obstetrics in the Department of Obstetrics and Gynecology, Xuanwu Hospital of Capital Medical University from January 2014 to June 2019 were recruited. Criteria included age 20-45 years, singleton pregnancy, previously healthy, no medical or surgical complications or immunosuppressive diseases, and not taking any immunosuppressive drugs. Cervical liquid-based cytology and HPV DNA detection were performed. Patients with abnormal results were further examined by colposcopy and a biopsy was obtained for pathological examination; cervical exfoliated cells were also collected and stored for assessing HR-HPV E6/E7 mRNA fragments.

All procedures performed in this study involving human participants were in accordance with the Declaration of Helsinki (as revised in 2013). The study was approved by the ethics board of Xuanwu Hospital of Capital Medical University (No. Linjishen[2014]001) and informed consent was taken from all the patients.

\section{Inclusion criteria}

(I) TCT (thinprep cytologic test) results are twice that of ASCUS with positive HR-HPV DNA test;

(II) TCT result is positive with or without HR-HPV DNA above ASCUS;

(III) HPV DNA test results are positive for HPV DNA types 16 and 18 .

\section{Exclusion criteria}

(I) Refusal to sign the informed consent form;

(II) Refusal to undergo colposcopy and cervical biopsy.

\section{Sample collection and processing}

\section{Collection and processing of TCT samples}

The woman was placed in the bladder lithotomy position, 
the cervix was fully exposed, a special cervical cell harvester (TCT) was placed at the junction of the cervical squamous column, and rotated clockwise around the cervix for 5-7 weeks to collect cells from the mouth and neck of the cervix. The exfoliated cells were placed in a vial containing CytoRich TCT preservation solution and stored at $4{ }^{\circ} \mathrm{C}$. Samples were labeled with the subject's identification (ID) number, and the obstetrician and gynecologist supplied the following data on the cytology request form: sampling date, ID number, name, age, last menstruation, past gynecological medical history, etc. After the samples were sent to the pathology department, two cytopathologists who were unaware of the HPV test results made a diagnosis according to The Bethesda System (TBS) recommended by the 2001 International Cancer Society (NCI). If there was no consensus, a third cytopathology expert assisted in distinguishing the diagnosis. The reported results comprised: within normal range (WNL), atypical squamous epithelial cells with unclear diagnostic significance (ASCUS), atypical squamous epithelial cells with highgrade disease (ASC-H), atypical glandular cells (AGC), lowgrade squamous intraepithelial lesion (LSIL), high-grade squamous intraepithelial lesion (HSIL), squamous cell carcinoma (SCC) and adenocarcinoma (Ade Ca).

In this study, cytological abnormalities were defined as ASCUS, ASCUS and above, and AGC at all levels.

\section{HPV DNA sample collection method and processing}

Insert the DNA-specific cervical cell collector (DNAPap, Digene) into the cervical canal for $1-1.5 \mathrm{~cm}$, rotate it clockwise for 3-5 weeks, and put the collection brush into a vial containing DNA preservation solution. Rapid flow hybridization gene chip technology was used in combination with low-density gene chip technology (HPV GenoArray Test kit, HybriBio Ltd., Hong Kong) to perform HPV DNA typing of specimens. This method uses HPV DNA PCR amplification and HybriBio's patented hybridization technology, which can simultaneously identify $21 \mathrm{HPV}$ genotypes, including 15 high-risk subtypes: HPV-16, -18 , $-31,-33,-35,-39,-45,-51,-52,-53,-56,-58,-59,-66,-68$ and 6 low-risk types $(-6,-11,-42,-43,-44$ and -CP8304).

\section{Colposcopy sample collection and processing}

Subjects underwent colposcopic examination, if the following conditions existed: the TBS result was ASCUS with two HR-HPV DNA positive tests; or ASCUS or higher with or without HR-HPV DNA if the test was positive; or HPV 16/18 genotype. Informed consent for electronic colposcopy was obtained from the subject who was prohibited from sexual activity, vaginal inspections, and vaginal drug application for 3 days before the examination. The optical electronic digital colposcopy (LEISEGANG, Germany) was operated and inspected by two professionally-trained and experienced gynecologists in strict accordance with the operating procedures of electronic colposcopy. The pathological results were interpreted jointly without knowing the HPV DNA test or TCT results, with a third superior expert to assist in distinguishing the diagnosis if there was no consensus. Pathological examination results were divided into normal or inflammation, cervical intraepithelial neoplasia (CIN) I, II or III, and invasive cervical carcinoma (ICC).

\section{HR-HPV E6/E7 mRNA sample collection method and processing}

Exfoliated cervical cells were collected from the subjects who underwent colposcopy in the meanwhile, for detection of HPV-16, -18, -31, -33, -35, -39, -45, -51, -52, -56, -58, $-59,-68$ and other 13 high-risk subtypes of HPVE6/E7 mRNA fragments. The detection was done with the Quanti Virus ${ }^{\circledR} \mathrm{HPV}$ E6/E7mRNA diagnosis kit. In this study, HR-HPV E6/E7 mRNA copy number $\geq 1$ copy/mL was considered positive.

\section{Quality control}

(I) A dedicated person was assigned for the collection and transportation of specimens. All liquid-based cytology specimens and pathological sections were read by two experts from the Department of Pathology, Xuanwu Hospital of Capital Medical University, and the diagnosis was made through joint consultation. If the diagnosis was in doubt, a third pathologist was invited to assist in the diagnosis. If the diagnosis was still in doubt, the section was sent to the Institute of Cancer Research of the Chinese Academy of Sciences for further consultation.

(II) All HPV DNA specimens were tested by the PCR Testing Center located in the Central Laboratory of Xuanwu Hospital of Capital Medical University. Unified kits (HybriBio Biotech) were used to strictly implement laboratory quality control standards and avoid cross-contamination.

(III) All pathologists and laboratory technicians involved in the testing had no access to relevant clinical data of the research samples. 
Table 1 Comparison of HPV DNA detection, HPV E6/E7 mRNA detection and pathological examination results during pregnancy

\begin{tabular}{|c|c|c|c|c|c|c|c|}
\hline Cervical lesion & Cases, $\mathrm{n}$ & \multicolumn{2}{|c|}{ HPV DNA } & \multicolumn{2}{|c|}{ HPV E6/E7 mRNA } & $\chi^{2}$ & $\mathrm{P}$ \\
\hline Normal or inflammation & 21 & $6(28.6)$ & $15(71.4)$ & $13(61.9)$ & $8(38.1)$ & 4.709 & 0.030 \\
\hline CIN I & 34 & $6(17.6)$ & $28(82.4)$ & $17(50.0)$ & $17(50.0)$ & 7.950 & 0.005 \\
\hline CIN II & 18 & $2(11.1)$ & $16(88.9)$ & $7(38.9)$ & $11(61.1)$ & 3.704 & 0.054 \\
\hline Total CIN & 63 & $9(14.3)$ & $54(85.7)$ & 27 (42.9) & $36(57.1)$ & 11.058 & 0.001 \\
\hline Total & 84 & $15(17.8)$ & $69(82.1)$ & $40(47.6)$ & $44(52.4)$ & 15.423 & 0.000 \\
\hline
\end{tabular}

CIN, cervical intraepithelial neoplasia; HPV, human papillomavirus.

\section{Data collation and statistical analysis}

SPSS 19.0 software was used for data sorting and statistical processing. The count data are expressed as a percentage, and the Pearson Chi-square test was used to compare the statistical significance of the difference. $\mathrm{P}<0.05$ was used as the statistical significance standard. Sensitivity, specificity and predictive values were calculated using contingency tables, and the $95 \%$ confidence interval (95\% CI) was calculated using the exact binomial method. Evaluation of the efficacy of HR-HPV E6/E7 mRNA detection and HPV DNA detection for high-grade cervical lesions was performed using receiver operating characteristic (ROC) curves.

\section{Results}

\section{Demographic characteristics}

This study investigated a total of 1,196 healthy singleton pregnant women aged between 20 and 45 years. There were 138 women lost to follow-up, so the final number enrolled was 1,058 . Among them, 322 were in the first trimester, 374 were in the second trimester, and 362 were in the third trimester. The average age of participants was $29.8 \pm 3.3$ years.

\section{Comparison of HPV DNA detection, HR-HPV E6/E7 $m R N A$ detection and patbological examination results during pregnancy}

Among the 1,058 pregnant study subjects, 118 had abnormal cytology results and/or were positive for HPV-16 or -18 subtypes DNA, and agreed to undergo colposcopy from which we successfully obtained biopsy pathology results for 84. The HR-HPV E6/ E7 mRNA colposcopy samples included 21 cases of normal or inflammatory cervical cells, 34 cases of CIN I, 18 cases of CIN II, 11 cases of CIN III, and 0 cases of ICC. The positive rate of HRHPV E6/E7 mRNA in the overall CIN and above was $57.1 \%$, which was significantly lower than the HPV DNA test of $84.1 \%\left(\chi^{2}=11.058, \mathrm{P}<0.001\right)$. Among those with normal or inflammatory results, the positive rates of HRHPV E6/E7 mRNA detection were $50.0 \%$ and $38.1 \%$, respectively, which were significantly lower than with HPV DNA detection $\left(\chi^{2}=7.950,4.709, \mathrm{P}=0.005,0.03\right)$. For the test results of CIN II and III, there was no significant statistical difference in the positive rate between the two. HR-HPV E6/E7 mRNA detection was the same as HPV DNA detection, both of which increased with the severity of cervical lesions, and the positive rate increased. There were significant statistical differences between groups $\left(\chi^{2}=19.834\right.$, $\mathrm{P}<0.001)$. The different detection results are shown in Table 1 .

\section{Comparison of diagnostic efficacy of HR-HPV DNA test and HR-HPV E6/E7 $m R N A$ test for high-grade cervical lesions during pregnancy}

Cervical lesions were defined based on CIN II, and those classified as CIN II or III were defined as high-grade cervical lesions; those below CIN II including CIN I and normal or inflammation were defined as low-grade cervical lesions and normal. The results of HPV DNA and HPV E6/E7 mRNA detection and analysis are shown in Table 2.

As shown in Table 3, the calculated sensitivity of HPV DNA to detect high-grade lesions was $86.2 \%$, the specificity was $21.8 \%$, the positive predictive value (PPV) was $37.1 \%$, and the negative predictive value (NPV) was $75 \%$. The sensitivity of HPV E6/E7 mRNA to detect high-grade lesions was $65.5 \%$, specificity of $54.5 \%$, PPV of $43 \%$, and 
Table 2 Comparison of HPV DNA and HPV E6/E7 mRNA detection for different grades of cervical lesion during pregnancy

\begin{tabular}{|c|c|c|c|c|c|}
\hline Cervical lesion & Total & \multicolumn{2}{|c|}{ HPV DNA } & \multicolumn{2}{|c|}{ HPV E6/E7 mRNA } \\
\hline Low-grade disease and normal ${ }^{*}$ & 55 & $12(21.8)$ & $43(78.2)$ & $30(54.5)$ & $25(45.5)$ \\
\hline High-grade lesions ${ }^{\star \star}$ & 29 & $3(10.3)$ & $26(89.7)$ & $10(34.5)$ & $19(65.5)$ \\
\hline total & 84 & 15 & 69 & 40 & 44 \\
\hline
\end{tabular}

${ }^{*}$, low-grade cervical lesions and normal: CIN I and normal or inflammation; ${ }^{* *}$, high-grade cervical lesions: CIN II and III. CIN, cervical intraepithelial neoplasia; HPV, human papillomavirus.

Table 3 Comparison of diagnostic efficacy of HPV DNA testing and HPV E6/E7 mRNA testing for high-grade cervical lesions during pregnancy

\begin{tabular}{lccc}
\hline Observation index & HPV DNA & HPV E6/E7 mRNA & $\chi^{2}$ \\
\hline Sensitivity (\%) & $89.7(26 / 29)$ & $65.5(19 / 29)$ & 4.858 \\
Specificity (\%) & $21.8(12 / 55)$ & $54.5(25 / 55)$ & 6.028 \\
PPV (\%) & $37.7(26 / 69)$ & $43.0(19 / 44)$ & 0.009 \\
NPV (\%) & $80.0(12 / 15)$ & $75.0(25 / 40)$ & 0.339 \\
\hline
\end{tabular}

Sensitivity $=$ number of true positive cases/(number of true positive cases + number of false negative cases) $\times 100 \%$. Specificity $=$ number of true negative cases/(number of true negative cases + number of false positive cases) $\times 100 \%$. (PPV = number of true positive cases/(number of true positive cases + number of false positive cases) $\times 100 \%$. NPV $=$ number of true negative cases/(number of true negative cases + number of false negative cases) $\times 100 \%$. HPV, human papillomavirus; PPV, positive predictive value; NPV, negative predictive value.

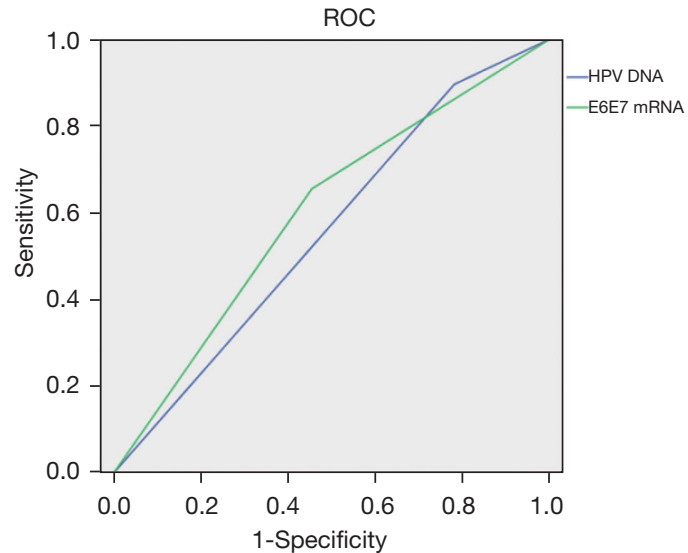

Figure 1 The ROC curves of HPV DNA and HPV E6/E7 mRNA detection during pregnancy for the diagnosis of high-grade cervical lesions. ROC, receiver operating characteristic; HPV, human papillomavirus.

\section{NPV of $75 \%$.}

Using the McNemar paired chi-square test, the sensitivity of HPV E6/E7 mRNA detection of high-grade cervical lesions was significantly lower than that of HPV DNA detection $\left(\chi^{2}=4.858, \mathrm{P}=0.028\right)$; the specificity of
HPV E6/E7 mRNA to detect high-grade cervical lesions was significantly higher than that of HPV DNA detection $\left(\chi^{2}=6.883, P=0.009\right)$. No significant difference was found between the PPV and NPV of HPV E6/E7 mRNA and DNA to detect high-grade cervical cancer.

\section{ROC curve of HR-HPV DNA detection and HR-HPV E6/ E7 mRNA detection during pregnancy for the diagnosis of bigh-grade cervical lesions}

As shown in Figure 1, the area under the ROC curves of HPV E6/E7 mRNA and DNA for detecting high-grade cervical lesions is 0.600 (95\% CI: $0.473-0.727$ ) and 0.557 (95\% CI: 0.431-0.684), respectively. The area under the curve of both diagnoses was $<0.7$, which indicated low accuracy, and the difference between the two was not statistically significant $(\mathrm{P}=0.389)$.

\section{Evaluation of HR-HPV E6/E7 mRNA detection for positive results of HR-HPV DNA detection}

Among the 69 subjects who tested positive for HR-HPV DNA, their HR-HPV E6/E7 mRNA levels were evaluated (Tables 4,5). The detection of HR-HPV E6/E7 mRNA 
Table 4 Assessment of HR-HPV E6/E7 mRNA ratio among HR-HPV DNA positive cases

\begin{tabular}{lccc}
\hline Variable & No. of HR-HPV DNA pos. cases & No. of mRNA pos. cases (\%) & No. of mRNA neg. cases (\%) \\
\hline Normal or inflammation & 15 & $4(26.7)$ & $11(73.3)$ \\
CIN I & 28 & $6(21.4)$ & $22(78.6)$ \\
CIN II & 16 & $11(68.8)$ & $5(31.2)$ \\
CIN III and above & 10 & $7(70.0)$ & $3(30.0)$ \\
Total & 69 & 28 & 41 \\
\hline
\end{tabular}

\#, evaluation of HR-HPV E6/E7 mRNA detection for positive results of HR-HPV DNA detection. CIN, cervical intraepithelial neoplasia; HPV, human papillomavirus.

based on a positive HPV DNA test increased the specificity of CIN II from $19.7 \%$ to $67.9 \%$, and the specificity of CIN III and above from $19.2 \%$ to $64.4 \%$. For cervical cancers above CIN II, the detection specificity of high-grade lesions increased from $21.8 \%$ to $76.7 \%$, and the differences were all statistically significant. Although the sensitivity decreased slightly, there was no statistical difference. In the diagnosis of high-grade cervical lesions, the specificity, PPV, and NPV of HPV DNA detection combined with HR-HPV E6/E7 mRNA detection showed improvement, and the specificity and PPV were statistically different $(\mathrm{P}<0.05)$.

\section{Postpartum outcomes of high-grade cervical lesions and predictive evaluation of the outcome of HR-HPV E6/E7 $m R N A$ detection}

Postpartum follow-up was performed for patients diagnosed with CIN II and above lesions. In 18 cases of CIN II, colposcopy and histopathological examination were performed again at 2-3 months after delivery. There was 1 case of progression to CIN III, 6 cases of maintained CIN II status, and 11 cases of reversion to CIN I and below. The progression rate was $5.6 \%(1 / 18)$, the stability rate was $33.3 \%(6 / 18)$, and the reversal rate was $61.1 \%(11 / 18)$. Among the 11 cases of CIN III, there were 4 cases of maintained CIN III status, and 7 cases of reversal to CIN II and below. The stability rate was $36.4 \%$ (4/11), and the reversal rate was $63.6 \%(7 / 11)$. No case progressed to ICC. Five cases of CIN III were all positive for HR-HPV E6/E7 mRNA during pregnancy. Of the 6 cases of maintained CIN II status, 4 cases were positive for HR-HPV E6/E7 mRNA during pregnancy, and 2 were negative. Of the 18 reversal cases, 10 were positive and 8 were negative. Among the cases of maintenance or progression of cervical lesions, the positive rate of HR-HPV E6/E7 mRNA detection during pregnancy accounted for $81.8 \%$ (9/11).

\section{Discussion}

In 2019, the consensus on the management of abnormal cervical cancer screening results and precancerous lesions in the ASCCP (American Society of Colposcopy \& Cervical Pathology) proposed a precise management model based on HPV screening combined with patient risk assessment, thereby making recommendations for diagnosis, treatment, and follow-up more personalized (10). The incidence of CIN during pregnancy ranges from $0.08 \%$ to $5.0 \%$ in the literature (11-13). Wu et al. reported that the incidence of abnormal cervical epithelial cells during pregnancy in Beijing was $3.95 \%$, which was similar to the incidence rate of $3.22 \%$ during non-pregnancy (14). During pregnancy, it is easy to cause bleeding during colposcopy because of ectopic cervical columnar epithelium, the tissue is thin, and capillaries are abundant. In severe cases, local cervical sutures are needed for hemostasis, which increases cervical damage. In addition, pregnant women are often resistant to vaginal operations and unwilling to undergo examinations, resulting in missed diagnosis of cervical lesions, especially malignant cervical lesions, which will ultimately affect the safety of both mothers and children.

The latest research suggests that HPV infection during pregnancy can be transmitted from the mother to the offspring through the vagina, increasing the risk of lung cancer in the offspring (15). However, because the current specificity of HPV DNA testing is not yet satisfactory, relying solely on a positive HPV test result can cause both psychological and economic stress to the pregnant woman during the perinatal period, which can also indirectly affect the safety of mother and the baby. The characteristics of cervical lesions during pregnancy make it necessary to avoid 


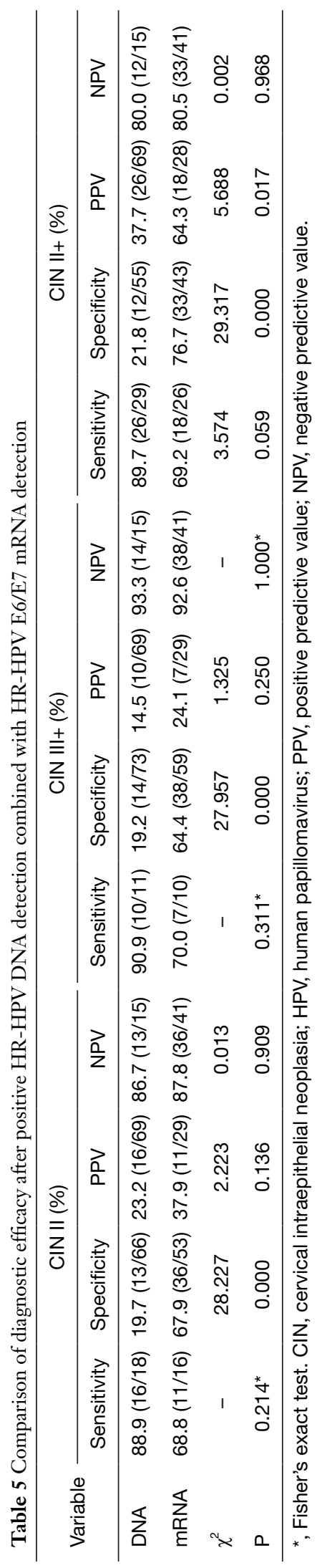

excessive diagnosis and treatment but at the same time, the diagnosis of cervical carcinoma should not be missed. Therefore, there is an urgent need for a detection method with good diagnostic performance that can effectively diagnose high-grade cervical lesions and minimize the missed diagnosis rate.

Many researchers have conducted studies of triage methods for HR-HPV-positive women, including classification based on cervical cytology to detect HPV-16/18 virus, detection of HPV-16/-18/-31/-33/-45 high-risk virus, and p16 or Ki67 immunohistochemistry (16).

After persistent infection of HR-HPV, under certain conditions, the $\mathrm{E} 6$ and $\mathrm{E} 7$ oncogenes are transcribed into E6 and E7 mRNA, and finally translated into E6 and E7 oncoproteins, which act on host cells and exert carcinogenic effects. Transcriptional expression of E6/E7 mRNA is the only pathway for the occurrence and development of cervical malignant lesions, and it is also a sign of HPV carcinogenic activity. Therefore, viral replication and transcription detection can be used to predict the risk of lesion progression.

Studies have shown that the sensitivity of non-pregnancy HPV DNA detection for the diagnosis of CIN II and above lesions is better than that of HPV E6/E7 mRNA detection ( 70-100\%), while the sensitivity of HPV E6/E7 mRNA detection is generally 60-90\%; however, HPV E6/E7 mRNA detection is more specific than HPV DNA detection $(5,17,18)$. Especially for predicting the progression of cervical lesions, it is better than HPV DNA testing $(19,20)$. However, there is a lack of reports on the use of HR-HPV E6/E7 mRNA detection to diagnose cervical lesions during pregnancy.

As the rate of CIN II and above lesions progressing to ICC has greatly increased, we took high-grade cervical lesions as the research object to evaluate the diagnostic efficacy of HR-HPV E6/E7 mRNA detection for CIN II and above lesions. Our results suggested that the positive rate of HR-HPV E6/E7 mRNA detection in the overall CIN and above during pregnancy was lower than that of HR-HPV DNA detection, and there was a significant statistical difference between the two methods. In CIN I and normal or inflammatory results, the positive rate of HRHPV E6/E7 mRNA detection was lower than that of HRHPV DNA detection, while for the results of CIN II and III, the positive rate of the two methods was not significantly different. HR-HPV E6/E7 mRNA detection is the same as HR-HPV DNA detection, both of which increased with the severity of cervical lesions, and the positive rate increased. 
We considered that the low positive rate of HR-HPV E6/ E7 mRNA detection for early cervical lesions may be due to the relatively silent period of the virus. Because most cases of CIN I can resolve, it is likely the cervical cells remain in the integration phase of HPV infection and do not progress to the E6/E7 oncogenes being highly transcribed and replicated, so the positive detection rate is low.

Compared with the detection sensitivity and specificity of HR-HPV E6/E7 mRNA detection for high-grade cervical lesions and that of HR-HPV DNA detection, we found that the specificity of HR-HPV E6/E7 mRNA detection was higher, but the sensitivity was lower. The results were consistent with other no pregnancy studies. There was no significant difference between the PPV and NPV of the two detection methods. In addition, there was no statistical difference between the area under the ROC curve of HR-HPV E6/E7 mRNA detection and HR-HPV DNA detection for the diagnosis of high-grade cervical lesions. The area under the ROC curve was $<0.7$, indicating insufficient diagnostic efficiency. Based on our finding, HPV DNA or HR-HPV E6/E7 mRNA detection alone is not effective screening tools for detecting high-grade cervical lesions during pregnancy.

Our preliminary data suggested that the use of HRHPV E6/E7 mRNA detection for HR-HPV DNA positive cases can significantly improve the diagnostic specificity of high-grade cervical lesions graded CIN II and above, and the specificity can be increased from $19.2-21.8 \%$ to $64.4-76.7 \%$. At the same time, the effect on sensitivity was not significant. Therefore, consider whether to apply the HR-HPV E6/E7 mRNA test in cases of TCT result of normal or ASCUS and LSIL, and the HR-HPV DNA test is positive (especially HPV-16 or -18 positive), as part of the risk assessment. Perform triage for colposcopy to achieve more accurate and individualized diagnosis and treatment.

Our study suggested that HR-HPV E6/E7 mRNA detection will reduce overscreening and treatment in women with occult HPV infection during cervical lesions screening follow-up, and is more suitable for triage in ASCUS and LSIL women. The level of HR-HPV E6/ E7 mRNA can be used as a surrogate in the screening and triage management of HR-HPV positive cases during pregnancy, in order to achieve the purpose of personalized management.

\section{Acknowledgments}

Funding: None.

\section{Footnote}

Reporting Checklist: The authors have completed the STARD reporting checklist. Available at https://dx.doi. org/10.21037/atm-21-4026

Data Sharing Statement: Available at https://dx.doi. org/10.21037/atm-21-4026

Conflicts of Interest: All authors have completed the ICMJE uniform disclosure form (available at https://dx.doi. org/10.21037/atm-21-4026). The authors have no conflicts of interest to declare.

Ethical Statement: The authors are accountable for all aspects of the work in ensuring that questions related to the accuracy or integrity of any part of the work are appropriately investigated and resolved. All procedures performed in this study involving human participants were in accordance with the Declaration of Helsinki (as revised in 2013). The study was approved by the ethics board of Xuanwu Hospital of Capital Medical University (No. Linjishen[2014]001) and informed consent was taken from all the patients.

Open Access Statement: This is an Open Access article distributed in accordance with the Creative Commons Attribution-NonCommercial-NoDerivs 4.0 International License (CC BY-NC-ND 4.0), which permits the noncommercial replication and distribution of the article with the strict proviso that no changes or edits are made and the original work is properly cited (including links to both the formal publication through the relevant DOI and the license). See: https://creativecommons.org/licenses/by-nc-nd/4.0/.

\section{References}

1. Woodman CB, Collins SI, Young LS. The natural history of cervical HPV infection: unresolved issues. Nat Rev Cancer 2007;7:11-22.

2. Mu L, Miao JR, Song JH. The correlation of HPV16 and HPV18 with local vaginal immunity after the treatment of cervical intraepithelial neoplasia. Transl Cancer Res 2020;9:4212-23.

3. Yang, X., Y. Cheng, and C. Li, The role of TLRs in cervical cancer with HPV infection: a review. Signal Transduct Target Ther, 2017. 2: p. 17055.

4. zur Hausen H. Papillomaviruses and cancer: from 
basic studies to clinical application. Nat Rev Cancer 2002;2:342-50.

5. Derbie A, Mekonnen D, Woldeamanuel Y, et al. HPV E6/ E7 mRNA test for the detection of high grade cervical intraepithelial neoplasia (CIN2+): a systematic review. Infect Agent Cancer 2020;15:9.

6. Kwan TT, Cheung AN, Lo SS, et al. Psychological burden of testing positive for high-risk human papillomavirus on women with atypical cervical cytology: a prospective study. Acta Obstet Gynecol Scand 2011;90:445-51.

7. Zhao X, Cui Y, Jiang S, et al. Comparative study of HR HPV E6/E7 mRNA and HR-HPV DNA in cervical cancer screening. Zhonghua Yi Xue Za Zhi 2014;94:3432-5.

8. Lv T, Wu Y, Liao Q, et al. Significance of high-risk human papillomavirus E6/E7 mRNA in cervical cytology ASCUS/ LSIL/ASC-H shunt. Progress in Modern Obstetrics and Gynecology 2017;26:670-3.

9. Liu J, Yang T, Hu Y, et al. The value of HPV E6/E7 mRNA quantitative analysis in distinguishing high-grade cervical squamous intraepithelial lesions from low-grade cervical squamous intraepithelial lesions. J Virol Methods 2021;289:114014.

10. Perkins RB, Guido RS, Castle PE, et al. 2019 ASCCP Risk-Based Management Consensus Guidelines for Abnormal Cervical Cancer Screening Tests and Cancer Precursors. J Low Genit Tract Dis 2020;24:102-31.

11. Stonehocker J. Cervical cancer screening in pregnancy. Obstet Gynecol Clin North Am 2013;40:269-82.

12. Wu YM, Wang T, He Y, et al. Clinical management of cervical intraepithelial neoplasia in pregnant and postpartum women. Arch Gynecol Obstet 2014;289:1071-7.

13. Tang $M$, You Z. Research progress in diagnosis and

Cite this article as: Yang YM, Wang SJ, Wang FY, Chen R, Xiao Q, Kang N, Liao QP. Preliminary study of the use of E6/E7mRNA detection in screening and triage management of HR-HPV infection during pregnancy. Ann Transl Med 2021;9(18):1428. doi: 10.21037/atm-21-4026 treatment of cervical precancerous lesions during pregnancy. Chinese Journal of Obstetrics and Gynecology 2020;55:420-4.

14. Wu X, Huang X, Zhang W. A clinical study of liquidbased cytology in screening pregnant women for cervical lesions. Chinese Journal of Obstetrics and Gynecology 2006;41:689-92.

15. Arakawa A, Ichikawa H, Kubo T, et al. Vaginal Transmission of Cancer from Mothers with Cervical Cancer to Infants. N Engl J Med 2021;384:42-50.

16. Ma X, Lakshmipriya T, Gopinath SCB. Recent Advances in Identifying Biomarkers and High-Affinity Aptamers for Gynecologic Cancers Diagnosis and Therapy. J Anal Methods Chem 2019;2019:5426974.

17. Zhu Y, Ren C, Yang L, et al. Performance of p16/Ki67 immunostaining, HPV E6/E7 mRNA testing, and HPV DNA assay to detect high-grade cervical dysplasia in women with ASCUS. BMC Cancer 2019;19:271.

18. Ren C, Zhu Y, Yang L, et al. Prognostic and diagnostic validity of p16/Ki-67, HPV E6/E7 mRNA, and HPV DNA in women with ASCUS: a follow-up study. Virol J 2019;16:143.

19. Cattani P, Siddu A, D'Onghia S, et al. RNA (E6 and E7) assays versus DNA (E6 and E7) assays for risk evaluation for women infected with human papillomavirus. J Clin Microbiol 2009;47:2136-41.

20. Baron C, Henry M, Tamalet C, et al. Relationship between HPV 16, 18, 31, 33, 45 DNA detection and quantitation and E6/E7 mRNA detection among a series of cervical specimens with various degrees of histological lesions. J Med Virol 2015;87:1389-96.

(English Language Editor: K. Brown) 